\title{
Jacques Réda tra le rovine di Parigi
}

\section{Federico Castigliano}

\section{(2) OpenEdition}

\section{Journals}

\section{Edizione digitale}

URL: http://journals.openedition.org/studifrancesi/3616

DOI: 10.4000/studifrancesi.3616

ISSN: 2421-5856

\section{Editore}

Rosenberg \& Sellier

\section{Edizione cartacea}

Data di pubblicazione: 1 décembre 2012

Paginazione: 523-535

ISSN: 0039-2944

\section{Notizia bibliografica digitale}

Federico Castigliano, «Jacques Réda tra le rovine di Parigi», Studi Francesi [Online], 168 (LVI | |II) | 2012, online dal 30 novembre 2015, consultato il 05 mars 2021. URL: http://journals.openedition.org/ studifrancesi/3616 ; DOI: https://doi.org/10.4000/studifrancesi.3616

\section{(c) (i) (9)}

Studi Francesi è distribuita con Licenza Creative Commons Attribuzione - Non commerciale - Non opere derivate 4.0 Internazionale. 


\section{Jacques Réda tra le rovine di Parigi ${ }^{1}$}

\section{La tradizione parigina della flânerie}

Che cosa resta, oggi, del flâneur parigino? Del passeggiatore solitario e osservatore dello spettacolo della città, nato in seno alla tradizione ottocentesca della littérature panoramique e delle pbysiologies ${ }^{2}$, e che divenne, secondo la definizione di Benjamin, una delle figure emblematiche della modernità urbana? Il flâneur non è il camminatore-filosofo - di cui possiamo trovare modelli letterari sin dall'antichità e, più recentemente, nelle promenades solitaires di Rousseau - che ricerca negli ambienti naturali lo stimolo alla meditazione e all'approfondimento dell'interiorità, ma un dandy dotato di una spiccata intelligenza critica e mosso da una curiosità insaziabile per lo spettacolo vario e mutevole della metropoli moderna. Nello "spazio libero" della capitale postrivoluzionaria il flâneur si distingue nettamente dalla folla: per il superiore status sociale che gli permette di «perdre son temps» e per la facoltà di interpretare lo scenario della città, divenuta ai suoi occhi un "testo" o un enigma da decifrare. L'interesse predominante per il mondo fenomenico e la libertà di movimento che caratterizzano il flâneur contrastano, da un lato, con i principi della tradizione metafisica e religiosa su cui si basava la civiltà preindustriale, e, dall'altro, con il dogma di produttività che sovrintende alla nascente società borghese. Di qui la natura paradossale del flâneur, specchio infranto della modernità: egli mette in scena la propria dissonante inattività ${ }^{4}$ proprio nel cuore pulsante della metropoli, e si cala nel tumulto della folla cercando di mantenere un distacco critico.

Sulla scorta della tradizione panoramica, Balzac aveva introdotto il flâneur, come personaggio letterario, nella Pbysiologie du mariage ${ }^{5}$, del 1829, mentre nella Physiologie du flâneur ${ }^{6}$, del 1841, Louis Huart lo aveva distinto dagli altri tipi umani che caratterizzavano Parigi, "capitale del XIX secolo". Secondo la Physiologie, il flâneur è riconoscibile per la sua eccentrica eleganza - cammina senza fretta, impeccabilmente vestito, portando «sa canne dans sa poche»-e per il suo atteggiamento ironico e

(1) Il presente articolo trae origine da alcuni incontri che ebbi con Réda nell'autunno 2009 e ripropone, con aggiunte e variazioni, idee sviluppate nei miei due lavori: Sept questions à Jacques Réda, «La Revue littéraire», 42, gennaio 2010, pp. 13-18 e Le divertissement du texte. Ecriture et flânerie chez Jacques Réda, «Poétique», 167, novembre 2010, pp. 461-476.

(2) La tradizione della littérature panoramique - termine con cui si usa indicare, a partire dagli studi di Walter Benjamin, l'insieme dei tableaux de moeurs e delle physiologies che descrivono la modernità parigina e i suoi personaggi rappresentativi - si sviluppò nella prima metà del XIX secolo sull'esempio del Tableau di Mercier. All'origine di questo genere vi è il fascino suscitato dal paesaggio architettonico e sociale della metropoli industriale, centro propulsore e "vetrina" del mondo moderno. Per lo studio di questo corpus letterario si faccia riferimento a W. Benjamin, Das Passagen-Werk, hrsg. von Rolf Tiedemann, Frankfurt am Main, Suhrkamp, 1982.

(3) Dictionnaire de l'Académie française, Paris, Institut de France, 1878, a.v.

(4) Si pensi alla condanna quasi inappellabile della flânerie pronunciata dal dizionario Larousse, di ispirazione positivista (P. LARousse, Grand Dictionnaire universel du XIX ${ }^{e}$ siècle, Paris 1872, a. v.).

(5) H. De Balzac, Physiologie du mariage, Paris, Levavasseur et Urbain Canel, 1829.

(6) L. Huart, Physiologie du flâneur, Paris, Aubert, 1841. 
denigrante rispetto ai concittadini. Essi si rincorrono tra loro affannati dalle faccende della quotidianità, mentre il flâneur si muove secondo il proprio gusto, «à droite ou à gauche sans raison, sans but», nutrendo il proprio sguardo dello spettacolo che lo circonda, ritornando volentieri sui propri passi «sans plus de motifs» ${ }^{7}$. La consacrazione letteraria del flâneur si ebbe due decenni più tardi con Baudelaire. In Le Peintre et la vie moderne $e^{8}$, egli rileva la necessità di una rinnovata pratica estetica che si adatti a un'inedita forma di bellezza, quella della città moderna, e individua nell'amico caricaturista Costantin Guy l'emblema dell'artista-flâneur capace di immergersi nella folla cittadina e vagare tra i «labyrinthes pierreux» della metropoli alla ricerca di un'improvvisa rivelazione da trasporre nelle sue opere d'arte. Il legame tra flânerie e scrittura presuppone, per Baudelaire, il ricorso a una nuova forma letteraria, il poème en prose - di cui fornisce egli stesso, con Le Spleen de Paris ${ }^{9}$, un modello di riferimento -, che si adatti alla frammentarietà delle esperienze della flânerie e alle subitanee intuizioni alla base della scrittura.

Elemento centrale della poetica baudelairiana, la metafora della città come testo $^{10}$ rinvia esplicitamente al ruolo del camminatore quale lettore e interprete della scena urbana. L'archetipo baudelairiano resterà vivo in forme ed esperienze letterarie anche distanti - dal romanzo realista alle avanguardie novecentesche - che, dalla seconda metà del XIX secolo, fanno riferimento al flâneur parigino per indicare una diversa tipologia di fruizione degli spazi della città. Gli autori surrealisti, in particolare, fecero della libertà di movimento e del principio di casualità un'attività artistica e un metodo di lavoro. Camminare senza meta è un'azione che svincola dal soggiogante imperativo del "produttivismo" e permette di sovvertire e rinnovare le percezioni della realtà, nel tentativo di riconciliare la vita quotidiana con gli impulsi e i desideri inconsci dell'individuo. Pur nutrito di clichés letterari della tradizione ottocentesca, il vagabondaggio dei surrealisti costituisce un allontanamento dall'idea originaria di $f \hat{a}-$ nerie. Se il flâneur di Huart era «l'homme le plus heureux de la terre» ${ }^{11}$, il passeggiatore surrealista, alla ricerca quasi affannosa di una rottura di ordine, o all'inseguimento di un chimerico alter ego femminile - come il Breton di Nadja o il Soupault di Les Dernières Nuits de Paris ${ }^{12}$ - sembra condannato a un'insoddisfazione permanente, a un indefinito inappagamento che è, forse, il presupposto essenziale per vivere nella dimensione dell'eterna possibilità.

$\mathrm{Fu}$ proprio la lettura dei surrealisti a ispirare Benjamin nei suoi studi sulla letteratura panoramica francese, un corpus di testi in cui il flâneur si distingue come una delle figure rappresentative della società parigina del XIX secolo. Benjamin pose l'accento sul valore critico del flâneur che, all'alba della modernità urbana, si caratterizza per un'ambiguità di fondo: in bilico tra la volontà borghese di emergere e il desiderio contraddittorio di svanire tra la folla e di cancellarsi. Nella seconda metà del Novecento alcuni movimenti d'avanguardia, come il Situazionismo, recuperarono i principi della flânerie surrealista, attribuendo alla camminata senza scopo (o dérive) un valore politico, in quanto forma di resistenza rispetto alle presunte "strategie di controllo" della società industriale. Proprio il confronto con l'archetipo ottocentesco divenne allora uno strumento per un'analisi delle trasformazioni che hanno interes-

(7) Ivi, p. 9 .

(8) Baudelaire, Le Peintre de la vie moderne, in Id., Euvres complètes, éd par C. Pichois, Paris, Gallimard, vol. II, 1991.

(9) Baudelaire, Le Spleen de Paris, in Id., Euvres complètes, vol. I, cit.

(10) Riconducibile ai Tableaux parisiens. Cfr.
K. Stierle, Der Mythos vos Paris, München, Hanser, 1993.

(11) Huart, Physiologie du flâneur, cit., p. 78.

(12) A. Breton, Nadja, Paris, Gallimard, 1964; P. Soupault, Les Dernières Nuits de Paris, Paris, Gallimard, 1997. 
sato la città contemporanea. Personaggio centrale anche nella riflessione sociologica e nel dibattito sugli sviluppi urbanistici - mi riferisco ad esempio ai lavori di Michel de Certeau, Keith Tester, Rebecca Solnit e Zygmunt Bauman ${ }^{13}$ - il flâneur è diventato una categoria critica autonoma, un riferimento per indicare una possibilità di esistenza nello spazio sociale della modernità, perdendo, almeno in parte, i lineamenti di una figura reale.

Oggi i termini flâneur e flânerie soffrono forse di un'eccessiva esposizione e corrono dunque il rischio di una svalutazione lessicale. Persino le agenzie turistiche e l'industria del divertimento li hanno assimilati per designare rituali di massa quali lo shopping, i viaggi organizzati o la passeggiata tra le nuove fantasmagorie del consumo. La libertà di movimento e lo sguardo critico, la volontà di interpretare lo spettacolo che si osserva - elementi su cui si basava la descrizione del flâneur ottocentesco - sono, tuttavia, difficilmente attribuibili al turista e al consumatore di oggi. Ed è indicativo che il flâneur, che trovava una propria definizione nella volontà di distinguersi dalla folla, sia entrato in crisi come personaggio letterario proprio nel momento in cui la pratica della flânerie sembra affermarsi come un fenomeno di massa. Ciò spiega anche perché il flâneur ottocentesco sia rimasto un riferimento per quegli autori che hanno fatto del rapporto tra individuo e città l'elemento centrale della loro opera. Il flâneur resta un simbolo per indicare un atteggiamento critico e contemplativo, rigorosamente individualista, rispetto alla realtà urbana.

I caratteri essenziali che definiscono la linea letteraria della flânerie parigina sono, in sintesi, la libertà di giudizio e di osservazione del camminatore, il tentativo di leggere e decifrare lo spazio urbano e la città stessa come testo, e la correlazione tra flânerie e scrittura. La corrispondenza di elementi tematici e scelte formali-come la ricorrenza del poème en prose - permette dunque di ipotizzare una linea che da Baudelaire giunge fino ai giorni nostri.

\section{Jacques Réda e la flânerie: rinascita di un modello letterario}

L'opera di Jacques Réda riflette con particolare evidenza l'eredità di questa tradizione $^{14}$. Con un accento d'ironia egli introduce la figura del flâneur nella città di oggi, in un contesto radicalmente mutato rispetto a quello in cui il personaggio era nato. L'appartenenza dell'autore alla linea letteraria che abbiamo tracciato è immediatamente segnalata dall'uso del poema in prosa e dalla ricorrenza di alcuni stereotipi e dai costanti richiami o omaggi ai luoghi comuni della tradizione. Della sua vasta produzione, che si sviluppa a partire dagli anni sessanta del Novecento, prenderò in esame quei testi che muovono appunto dal tema della flânerie urbana a Parigi. Si tratta per lo più di raccolte di prose poetiche, in cui la descrizione di scene urbane si alterna all'analisi delle forme d'interazione tra il passeggiatore e la citta ${ }^{15}$. L'esplorazione dello spazio urbano - che l'autore svolge a piedi, in bicicletta o in "solex" - è dunque il presupposto dell'attività letteraria. Si deve a ciò la struttura rapsodica e frammentaria delle sue raccolte, così come l'ampia gamma di soluzioni formali che caratterizzano

(13) M. DE CeRTEAu, L'invention du quotidien, Paris, Gallimard, 1990; K. TESTER The Flâneur, New York, Routledge, 1994; Z. Bauman, Postmodernity and Its Discontents, Oxford, Blackwell Publishers, 1997; R. SOLNIT, Wanderlust: a History of Walking, New York, Penguin, 2000.

(14) C. CoQUIO, Le retour éternel du flâneur, in Approches de Jacques Réda, textes réunis par C. Van
Rogger Andreucci, Pau, Publications de l'Université de Pau, 1994.

(15) Les Ruines de Paris (1977), Hors les murs (1982), Beauté suburbaine (1985), Recommandations aux promeneurs (1988), Le Sens de la marche (1990), La liberté des rues (1997), Le citadin (1998), Moyens de transport (2000), Accidents de la circulation (2001), Europes (2005). 
la scrittura, sempre fluttuante tra poesia e prosa. La sede prediletta delle flânerie di Réda - Parigi e le sue banlieues - lo pone immediatamente in contatto con la tradizione $^{16}$, dando vita a un interessante gioco di allusioni e richiami storico-letterari che nutre la sua scrittura. Il paesaggio parigino, prodotto di un' "invenzione" culturale, appare come una complessa trama di riferimenti che il flâneur prova a sciogliere:

Il serait à présent tentant de comparer la ville à un texte: un poème, un roman, une superposition de récits comme dans un palimpseste où rien ne s'effacerait complètement, où chaque nouvelle rédaction, née de la précédente, garderait des traces à mesure de plus en plus rares, mais d'autant plus parlantes, de tous les états successifs ${ }^{17}$.

Si tratta di una metafora ricorrente, quasi il segno di appartenenza alla tradizione che attribuisce al camminatore il ruolo di lettore e interprete dello spettacolo della città. Ma Réda è consapevole che la sovrapposizione di memorie storico-letterarie e vicende personali rende impossibile una lettura lineare del testo urbano, divenuto, appunto, un palinsesto che suggerisce possibili itinerari al camminatore, favorendo dunque la scrittura stessa. Questo legame profondo tra flânerie e attività letteraria è l'elemento centrale della poetica di Réda e trova eco in immagini di chiara ascendenza baudelairiana: «...toute la ville et son espace irrésolu I Ne font qu'un avec le réseau mobile mais fidèle | Des phrases que nos pas écrivent quand nous circulons» ${ }^{18}$.

Il richiamo alla figura del flâneur ottocentesco è dunque appropriata per spiegare sia le modalità attraverso cui Réda si relaziona agli spazi urbani, sia il rapporto che intercorre, nelle sue opere, tra flânerie e letteratura. Camminatore senza scopo, Réda misura lo scarto tra la propria vocazione di flâneur e altre forme di esplorazione della città orientate unicamente a un fine. In un frammento di La liberté des rues, egli prende le distanze da chi ha definito la flânerie come un moto che segue il principio di piacere connesso alla libera volontà del camminatore oppure, viceversa - si pensi all'esperienza dei situazionisti -, come un percorso guidato da scelte di politici o urbanisti che si riflettono sulla struttura stessa della città ${ }^{19}:$ «j'ai d'ailleurs cessé de croire que je circule au gré de ma fantaisie. Je ne pense pas davantage obéir, en circulant, à quelque plan préétabli pour me guider ou me perdre ${ }^{20}$. A orientare Réda è invece «une espèce de pendule, situé dans le même coin du cerveau que l'horloge qui me donne l'heure à la minute près» ${ }^{21}$. Metafora, questa, che riconduce alla tradizione «panoramica» ottocentesca, in cui era lo spettacolo della città a determinare l'itinerario del curioso flâneur. Da ciò il rifiuto, in Réda, d'ogni movimento che sia subordinato a un fine e la conseguente critica ai tentativi di classificare e misurare gli spazi della città, come invece teorizzato dagli autori dell'Oulipo:

Beaucoup s'enferment alors dans une petite magie algorithmique ou stochastique, fondée en général sur un comptage maniaque des pas, autorisant divers calculs de probabilités et prévisions de récurrences qui ne leur donnent que de façon illusoire le contrôle et l'intelligence de la situation ${ }^{22}$.

(16) «Le flâneur peut naître partout; il ne sait vivre qu'à Paris» (Paris ou Le livre des cent-et-un, Paris, Ladvocat, 1831, vol. VI, p. 95).

(17) J. RÉDA, La Liberté des rues, Paris, Gallimard, 1997, p. 70.

(18) RÉDA, La course, Paris, Gallimard, 1999, p. 11

(19) Mi riferisco, nel primo caso, agli autori surrealisti e, nel secondo, all'esperienza dell'avanguardia situazionista.

(20) RÉDA, La Liberté des rues, cit., p. 59.

(21) Ivi, p. 55.

(22) Ivi, p. 56. 
Attraverso la sistematica catalogazione delle forme del paesaggio parigino gli oulipiani hanno tentato d'imporre il rassicurante dominio della razionalità sulla scena varia e inquietante della metropoli contemporanea. La contraintre, alla base della scrittura degli oulipiani, ma anche della loro modalità di movimento nella città, fornisce un sistema matematico, una regola che permette di tenere sotto controllo un mondo altrimenti sfocato e angosciante. Tale tentativo di imprigionare lo sfaldamento della realtà attraverso il linguaggio assume una direzione contraria alla poetica di Réda, che si propone invece un'esaltazione del mondo fenomenico:

Et le vrai est que la liberté des rues ne se plie à aucun type de rangement; qu'il faut être inconscient ou présomptueux pour imaginer qu'elles coïncident avec nos répertoires, nos graphiques, notre alphabet; qu'elles se laissent impressionner par les noms et les numéros qu'on leur attribue ${ }^{23}$.

La camminata di Réda, disconnessa da qualsiasi funzione pratica, diviene un'azione "pura" e incondizionata, un rituale da compiere in solitudine ascetica, caricato di un forte valore simbolico. Come la creazione artistica, la flânerie è una forma di contemplazione e celebrazione del visibile e non richiede uno scopo o una giustificazione al di fuori di se stessa. Il flâneur, tutto teso nell'esercizio di osservazione dello spettacolo che gli si apre davanti, parla raramente di sé: i frammenti di Réda sono dunque prevalentemente descrittivi, caratterizzati da una scrittura digressiva, ricchissima di riferimenti e di particolari, che tende a mimare e accompagnare le divagazioni del camminatore. Sono le strade stesse a suggerire gli spostamenti e, al contempo, a dettare l'ispirazione allo scrittore: «Il me semble plutôt que sans se préoccuper de mon cas, ce sont les rues elles-mêmes qui se déplacent, s'ébattent - et je me laisse remuer, prenant discrètement ma part du plaisir qu'elles échangent ${ }^{24}$. E se, sostiene Réda, «il n'y a pas de différences entre ma façon d'écrire sur elles et leur comportement $\gg^{25}$, la letteratura stessa si costruisce, piuttosto che su una struttura predefinita, in base agli «accidents de la circulation ${ }^{26}$.

D'altra parte, se la modernità predilige la circolazione rapida, finalizzata a uno scopo pratico, la camminata del flâneur ${ }^{27}$ appare oggi anacronistica e sospetta. Réda si muove con il «pas furtif de l'hérétique» ${ }^{28}$, a piede libero «comme un bandit, comme ce réprouvé, là-bas ${ }^{29}$, in contraddizione rispetto ai principi che improntano la società contemporanea: «On constate que le trajet Paris-Lyon [...] a, durant ces dernières années, diminué de plus de moitié en temps. C'est une grande perte pour ceux dont le caractère, même dans la tension du voyage, demeure disposé à flâner» ${ }^{30}$. Proprio quando si ritrova a indossare gli abiti del flaneur, rivisitandone le formule e i topoi tradizionali, l'autore è consapevole di quanto ciò sembri paradossale e ridicolo. L'ironia di Réda ha quindi una valenza duplice: presa di distanza rispetto alla vita moderna, ma anche nei confronti del proprio personaggio letterario e al ruolo che egli prova a incarnare. Gli stereotipi della tradizione sono quindi evocati con un intento parodico: i passages ad esempio, luoghi emblematici della flânerie ottocentesca, appaiono all'autore come un prosaico susseguirsi di ristoranti e parrucchieri ${ }^{31}$, mentre all'errare notturno del flâneur viene attribuito, scherzosamente, una giustificazione mistica:

(23) Ibid.

(24) Ivi, p. 59

(25) Ivi, p. 60

(26) Cfr. RÉDA, Accidents de la circulation, Paris, Gallimard, 2001.

(27) «sans hate» (RÉDA, Les Ruines de Paris, Paris, Gallimard, 1993, p. 56).
(28) Ivi, p. 9.

(29) RÉDA, Hors les murs, Paris, Gallimard, 1982, p. 43.

(30) RÉDA, Recommandation aux promeneurs, $\mathrm{Pa}$ ris, Gallimard, 1988, p. 86.

(31) RÉDA, Le Citadin, Paris, Gallimard, 1998, pp. $179-182$. 
«N'ayant plus une cigarette, | Je vais au hasard dans Paris | [...] Et voici le traiteur | Sur le point de fermer boutique. I Etes-vous acheteur? I Non, monsieur, ma quête est mystique» ${ }^{32}$. Allo stesso modo, in un lungo frammento delle Ruines de Paris, la maschera dell'esteta decadente che si distingue nell'acquisto di prodotti rari cade inesorabilmente nel confronto con la pratica massificata dello shopping, costringendo l'autore a ripiegare sull'autoironia:

Rue Saint-Honoré, trois paquets de cigarettes Leduc à la Civette. Je suis dans mon genre un expert d'une ampleur assez rare pour les tabacs. Longtemps pantouflard et pusillanime, je me suis décidé aux voyages presque uniquement dans le but de fumer. On me voit sans arrêt sortir des marques invraisemblables de toutes mes poches, et je finirai par savoir tousser en beaucoup de langues, mais peu de gens peuvent admettre que je recherche ainsi l'Absolu ${ }^{33}$.

Distinguersi dalla massa - ideale cui aspirava il flâneur di Huart, esperto conoscitore della città - è un'operazione destinata al fallimento. Il dandy è degradato a consumatore che "costruisce" la propria identità attraverso l'acquisto compulsivo di merce industriale, finché, avvilito e solo, «chacun retourne dans sa banlieue, encombré et comme moi hébété de paquets» ${ }^{34}$. L'ironia con cui l'autore prende le distanze dal proprio personaggio letterario trova spesso corrispondenza, a livello formale, nello scontro tra il lessico ricercato e letterario (anche applicato agli aspetti più prosaici del paesaggio metropolitano) con forme di uso più familiare: «Ils transbahutent leur malaise tels des déménageurs | L'air blasé, mais troublés par ce qui scintille et rutile: | vitrines et cinés, le ball flambant du King Burger» ${ }^{35}$.

\section{Il poème en prose tra antirealismo e rappresentazione della scena urbana}

Susanne Bernard, descrivendo il fenomeno del poema in prosa nella letteratura francese dal Gaspard de la Nuit di Bertrand fino agli anni cinquanta del Novecento ${ }^{36}$, individuò due principali linee di sviluppo all'interno di questo genere letterario. La prima, che corrisponde a un'esasperazione del principio distruttore e anarchico che presiede alla forma frammentaria, e dunque alla negazione di ogni istituzione formale preesistente, trova la sua espressione paradigmatica con le Illuminations di Rimbaud. La seconda, invece, dominata da un principio organizzatore che tende a dare un nuovo assetto al discorso letterario dopo la dispersione in frammenti, è presieduta da un'idea di ordine, di conciliazione, che si realizza nel testo con un processo di stilizzazione della realtà. Negli autori appartenenti al secondo gruppo - il cui archetipo è lo Spleen de Paris di Baudelaire - le percezioni sensibili e il dato biografico sono filtrati mediante un rigoroso controllo formale, con strumenti retorici quali la repetitio, la variatio, strategie metriche e musicali, simmetrie e corrispondenze che integrano $\mathrm{i}$ contenuti in una sequenza armoniosa.

Il poema in prosa di Réda, contraddistinto dalla ricerca di una compostezza formale quasi neoclassica e dal conseguente rifiuto di moduli espressionisti e modernisti, sembra avvicinarsi al secondo modello proposto dalla Bernard. Il ricorso a un lessico prezioso e spiccatamente letterario - non senza una nota d'ironia - l'utilizzo di strutture frastiche complesse, con il prevalere di soluzioni ipotattiche, conducono Réda a un esercizio di scrittura virtuosa, senza che si giunga mai, tuttavia, alla rottura

(32) RÉDA, Châteaux des courants d'air, Paris, Gallimard, 1986, p. 15.

(33) RÉDA, Les Ruines de Paris, cit., p. 66.
(34) Ivi, p. 72.

(35) RÉDA, Châteaux des courants d'air, cit., p. 47. 
della norma linguistica e letteraria. In linea di continuità con i principi che guidano il poème en prose baudelairiano, la scrittura di Réda trae la propria coerenza di poema da un andamento lirico e musicale, con una prosa ricchissima di figure di suono, e con soluzioni ritmiche che alludono più alla forma poetica che alla prosa. La ricerca nostalgica del verso è evidente quando l'autore adotta una prosa spezzata, la cui frase è interrotta dai frequenti "a capo". Del resto, lo stesso rigore e preziosismo formali contraddistinguono le poesie di Réda, incastonate nelle raccolte tra i diversi frammenti di prosa. Nelle poesie sono ricorrenti inversioni sintattiche, tournures e rime inconsuete, che danno luogo a un gioco formale particolarmente ardito nelle descrizioni del cielo parigino: «Car après la douceur de mars approchent les âpres Pâques | avec ces froissements sur le marbre où luit un ciel de fer. | Le soleil n'est plus guère qu'un mince comprimé d'aspirine | en train de se dissoudre dans le brouillard» ${ }^{37}$; «Mais tandis que l'orage éternue, I se reflète en roulant dans l'émail des camions | un soleil coléreux déjà bas sur Versailles ${ }^{38}$.

Il ricorso al modello di Bertrand sembra opportuno per spiegare come le scelte formali del poème en prose di Réda implichino un allontanamento dalla poetica del realismo. La sua scrittura non ha come scopo una restituzione "fotografica" della realtà, né vuole rappresentare - al pari della letteratura panoramica, da cui il personaggio del flâneur era nato - la complessità sociologica o urbanistica della Parigi contemporanea. A essere messa in scena è l'azione stessa della flânerie, mentre il paesaggio diviene oggetto della riscrittura dell'autore. Gli elementi formali che caratterizzano il poema in prosa di Réda sono quindi funzionali a una reinvenzione della realtà visibile, alla sua trasfigurazione sistematica, così che gli spazi urbani sono tradotti in una dimensione immaginaria. In un frammento di Les Ruines de Paris, place de la Concorde diviene un luogo addirittura marittimo:

Car il arrive qu'une obsession de transmutation urgente nous possède: à force de le contempler, passer du côté du spectacle, entrer dans la substance aveugle qui sait, qui resplendit. Comme si l'homme en arrêt momentané sur ses deux jambes, visant les cieux, ne débordait pas l'invincible enveloppement d'étoiles. C'est l'instant où je trébuche au bord des pelouses, dans les arceaux. Tant bien que mal enfin j'atteins la place de la Concorde. L'espace devient tout à coup maritime. Même par vent presque nul, un souffle d'appareillage s'y fait sentir. Et, contre les colonnes, sous les balustrades où veillent des lions, montent en se balançant des vaisseaux à châteaux du Lorrain, dont tout le bois de coque et de mâts, et les cordes, et les toiles sifflent et craquent, déchirant l'étendard fumeux qui sans cesse se redéploie au-dessus de la ville. Je vais donc comme le long d'une plage, par des guérets ${ }^{39}$.

Queste scelte formali accomunano Réda con i propri modelli e con gli autori che riconosce affini alla sua scrittura, quali il Mallarmé della prosa, il Claudel de La Connaissance de l'Est, Jean Follain, Julien Gracq e Charles-Albert Cingria ${ }^{40}$ : scrittori che uniscono l'attenzione per la città alla scelta di una prosa poetica. Follain in particolare, offre in Paris $(1935)^{41}$ un modello di reinvenzione e destrutturazione della città attraverso l'operazione della scrittura. A differenza del suo contemporaneo Fargue, che in Le Piéton de Paris ${ }^{42}$ riporta meticolosamente i dettagli pittoreschi o folkloristici

(36) S. BERNARD, Le poème en prose de Baudelaire jusqu'à nos jours, Paris, Nizet, 1959.

(37) RÉDA, Les Ruines de Paris, cit., p. 53.

(38) RÉdA, Beauté suburbaine, Périgueux, Fanlac, 1985, p. 11.

(39) RÉDA, Les Ruines de Paris, cit., pp. 9-10.

(40) Cfr. RéDA, Les Ruines de Paris, cit, p. 67. L'autore segnala questi modelli letterari in una recente intervista: F. CAStigliano, Sept questions à Jacques Réda, «La Revue littéraire», 42, janvier 2010.

(41) J. Follain, Paris, Paris, Édition Corrêa, 1935.

(42) L.-P. Fargue, Le Piéton de Paris, Paris, Gallimard, 1993. 
della capitale, Follain dà vita a una metamorfosi letteraria di Parigi, ne reinventa il paesaggio e lo ricompone attraverso il linguaggio del poema in prosa.

A partire dalla realtà urbana che osserva e attraversa, Réda costruisce dunque una struttura autonoma e conchiusa: quella del testo letterario. L'onnipresente accento ironico, sottolineato anche dall'uso di un registro linguistico ben diverso dalla parlata quotidiana, concorre ad allontanare lo stile di Réda da qualsiasi pretesa di oggettività o da un tentativo di scarna descrizione fenomenica del paesaggio urbano, come quello intrapreso da Perec in Tentative d'épuisement d'un lieu parisien o Espèces d'espaces ${ }^{43}$. Una riscrittura della città e del suo spettacolo che, subordinando l'oggettività delle descrizioni ai principi formali e poetici, afferma il primato del testo letterario sulla realtà:

J'écris de la prose - ou des vers - où se reflètent nécessairement des figures en mouvement du monde qui m'entoure, et je ne les rejette certes pas, mais je dois les intégrer à la langue que je me suis construite. Si l'écrivain est un promeneur [...] il est d'abord le promeneur et le site de sa propre langue ${ }^{44}$.

Tentativi di realismo e di riflessione sulle problematiche sociali della realtà urbana sussistono in autori recenti che hanno cercato di documentare gli aspetti critici della città contemporanea. Si pensi, per restare in ambito parigino, a Jean Rolin. Impegnato, in opere quali Zones (1995) o La Clôture (2002) ${ }^{45}$, nell'esplorazione di Parigi e delle sue banlieues, Rolin esegue una descrizione lucida e oggettiva degli spazi urbani, integrata nei suoi reportages narrati in prima persona da una raccolta di dati e fatti di cronaca ${ }^{46}$. La flânerie diviene un metodo di lavoro, uno strumento d'indagine $^{47}$ per perlustrare le zone marginali del territorio parigino, nell'intento di comprendere le dinamiche sociali e le linee di forza che caratterizzano l'intrigo etnico e culturale "postmoderno". Nella Parigi considerata come esempio di métissage o di uniformazione, Rolin mette in luce il riemergere in forma spesso violenta dell'identità e dell'idea di territorio, l'irriducibile differenza tra i vari gruppi etnici e culture, registrando la divisione dello spazio metropolitano in comunità distinte e zone demarcate. La prosa di Rolin, che pur procede per agglomerazione di frammenti, è essenzialmente denotativa e realistico-figurativa, priva di particolari effetti stilistici, poiché rimanda all'esperienza di giornalista e reporter "dal fronte" piuttosto che alla tradizione del poème en prose. Tra i molti esempi, ne cito uno in particolare, a testimonianza del suo stile scarno, improntato a un forte realismo, ricco di notazioni e precisi riferimenti spazio-temporali, elementi assenti nei testi di Réda:

Au lever du jour, l'enseigne de la tour Pleyel, bleue, tourne imperturbablement sur son axe. Les premiers immeubles à recevoir la lumière du soleil sont les tours de la rue Jean-Cocteau, et le premier commerce à ouvrir, porte de Clignancourt, est le kiosque à journaux situé en face du PMU. Puis, plus tard, le PMU lui-même. A 6 h 30, alors que déjà le soleil tape mais que la place est encore presque déserte, une grosse Peugeot noire marque un temps d'arrêt à l'entrée de la rue Letort... ${ }^{48}$

Altro esempio di realismo descrittivo che rimanda alla tradizione della flânerie, perché basato sulla sovrapposizione di esplorazione della città e scrittura frammen-

(43) G. Perec, Tentative d'épuisement d'un lieu parisien, Paris, Christian Bourgois, 1982 e PEREC, Espèces d'espaces, Paris, Galilée, 2000.

(44) F. Castigliano, Sept questions à Jacques Réda, cit., p. 18.

(45) J. Rolin, Zones, Paris, Gallimard, 1995; Ro-
LIN, La Clôture, Paris, Gallimard, 2002.

(46) Rolin, La Clôture, cit., p. 28.

(47) «La nuit des mes premières investigations» (Ivi, p. 50).

(48) Ivi, pp. 58-59. 
taria, è il Journal du debors ${ }^{49}$ (1993) di Annie Ernaux. In quest'opera l'autrice esplora uno spazio «aux limites incertaines» e «privé de toute mémoire» ${ }^{50}$, trascrivendo gesti e parole degli anonimi passanti che incontra nei treni suburbani, nei supermercati e nei centri commerciali di una "ville nouvelle" situata a 40 chilometri dal centro di Parigi. Ernaux alterna la registrazione di elementi visivi a trasposizioni di dialoghi e proprie riflessioni secondo una struttura che procede per tessere giustapposte. Ernaux e Rolin ambiscono a decifrare uno spazio inedito, quello delle "zones" e delle "villes nouvelles", teatro di alcuni terribili fatti di cronaca. Al centro urbano, percepito come la vetrina sfavillante del commercio, si oppone il degrado bestiale delle periferie a cui corrisponde un modello di integrazione o assimilazione dagli esiti assai dubbi. Il riferimento a questi autori è utile per misurare lo scarto e la posizione peculiare di Réda verso i problemi e le emergenze della città contemporanea. Anche Réda, che è stato soprannominato «le flâneur des banlieues» ${ }^{51}$, attraversa questi spazi, ma essi restano lo sfondo per il rito ascetico della flânerie. La libertà di movimento che caratterizza il flâneur implica un atteggiamento individualista e una libertà ideologica: una forma di disimpegno rispetto ai problemi sociali e politici della contemporaneità, cui corrisponde un grande impegno nei confronti della scrittura.

\section{L'insegnamento delle rovine}

Il tema delle rovine di Parigi risale, come noto, al Secondo Impero - si pensi al Paris démoli, mosä̈que de ruines (1853) di Eduard Fournier, o a Ruines de Paris (1858) di Charles Monselet ${ }^{52}$-, all'epoca dei "grands travaux" progettati e diretti da Haussmann, artista "distruttore", e occupa uno spazio rilevante nei Tableaux parisiens di Baudelaire. Karlheinz Stierle, nel suo studio su Parigi, nota che le demolizioni della città vecchia assumono per Baudelaire un valore simbolico. In Le Cygne, in particolare, lo spettacolo delle rovine diventa allegoria della caducità delle cose, mentre la corrispondenza tra paesaggio urbano e dimensione interiore dell'artista-flaneur ${ }^{53}$, già evidenziata da Benjamin, acquisisce un accento malinconico. Questa poesia, come prova la ricorrenza dell'espressione «Le vieux Paris n'est plus. La forme d'une ville | change plus vite, hélas, que le cœur d'un mortel!», è poi divenuta un riferimento comune a molti autori francesi - quali Gracq ${ }^{54}$, Debord, Roubaud e Réda - che hanno riconosciuto nelle metamorfosi del paesaggio urbano un riverbero delle proprie vicende personali. Un topos, insomma, per esprimere la consapevolezza, dolorosa per questi autori, del processo di trasformazione delle città e che riflette, almeno in parte, il paradosso che accompagna il flâneur sin dalle origini, tra esaltazione della modernità e atteggiamento sospettoso verso di essa.

Les Ruines de Paris, pubblicato nel 1977, evoca sin dal titolo il sentimento di perdita e frammentarietà che segna l'esperienza del camminatore odierno. Le rovine sono le vestigia del passato e le tracce della vita dell'autore, elementi del paesaggio

(49) A. Ernaux, Journal du dehors, Paris, Gallimard, 1993.

(50) Ivi, p. 7.

(51) C. Van Rogger Andreucci, Avant-propos, in Approches de Jacques Réda, cit., p. 7. Crf. anche RÉDA, La tourne, Paris, Gallimard, 1975, p. 151: «'explorerai des périphéries». L'autore ricerca una «beauté suburbaine» che si nasconde «hors les murs» (RÉDA, Hors les murs, Paris, Gallimard, 1982).

(52) E. Fournier, Paris démoli, mosäque de ruines, Paris, J. Dagneau, 1853; C. Monselet, Les ruines de Paris, Paris, L. de Potter, 1858.

(53) Cfr: B. Liu, Les Tableaux parisiens de Baudelaire, Paris, L'Harmattan, 2003.

(54) Su questo punto si potrebbe individuare una singolare affinità con un'opera di Gracq, di una quindicina di anni successiva alle Ruines de Paris, il cui titolo (La forme d'une ville) rievoca il testo di Le Cygne: «L'ancienne ville-l'ancienne vie - et la nouvelle se superposent dans mon esprit plutôt qu'elles ne se succèdent dans le temps» (J. Grace, La forme d'un ville, Paris, José Corti, 1985, p. 9). 
urbano che il flâneur ha introiettato o che, viceversa, sono divenute proiezioni della sua interiorità. Se il corpo della città documenta la stratificazione del tempo, le distruzioni intaccano direttamente la memoria dell'autore ${ }^{55}$. Raffrontando la Parigi presente con quella passata, la scrittura, chiamata in aiuto della memoria, fa riaffiorare tristemente ciò che non è più:

D'après le plan ancien que j'utilise, une Manufacture de Tabacs se tiendrait rue des Meuniers. Je comptais beaucoup sur cette manufacture. Il n'y en a plus. À la place, un de ces grands rayonnages d'habitation...

Le considerazioni di Réda, che percepisce il proprio anacronismo rispetto alla tradizione ottocentesca cui si ispira, indurrebbero a considerare il flâneur come un personaggio storico, relegato a un ambiente urbano ben definito - la Parigi del primo Ottocento - e la flânerie come un'attività impraticabile nella città odierna. «Un moment vient d'ailleurs où l'incessante modification de la ville affecte et peut décourager le promeneur $»^{57}$ : la città contemporanea, «amputée de sa mémoire ${ }^{58}$, usurpata dal «béton indifférent» ${ }^{59}$, è una «grisaille» ${ }^{60}$ satura di «maisons neuves pareillement froidement inhabitées» ${ }^{61}$, mentre l'uniformità e la dispersione spaziale ${ }^{62}$ dissolvono il piacere della flânerie: «Un jour, dans la globale conurbation de la planète métamorphosée en bouquet de bouquets de banlieues, il n'y aura plus vraiment de départs, plus vraiment d'arrivées...» $\gg^{63}$. L'avanzare della modernità - segnalato dalle «forêts de grues ${ }^{64}$ innalzate al cielo come vessilli che dominano il paesaggio spettrale delle rovine - cancella l'identità dei luoghi e per sostituirla con la fredda piattezza dell'uguale: «À l'angle de la rue de Vaugirard une petite boucherie, seul souvenir du faubourg ancien, et ça pourrait être autrement n'importe quel carrefour, n'importe quel immeuble....» ${ }^{65}$.

La rievocazione nostalgica della Parigi perduta è un tema che risale alla seconda metà del XIX secolo, un'epoca di radicale ristrutturazione del tessuto urbano e delle regole di vita della capitale, e che conosce una ripresa nel Novecento, dall'avanguardia surrealista ai situazionisti. Del resto alcune trasformazioni che hanno interessato la città del xx secolo, e in particolare in occasione della ricostruzione post-bellica, sono interpretabili come la realizzazione di un'ideologia che ha le sue origini proprio con Haussmann: un processo di privatizzazione, razionalizzazione e specializzazione degli spazi, attuato in nome delle crescenti necessità di spostamento di uomini e di merci, e delle sempre più elevate esigenze riguardanti l'igiene, la sicurezza e il controllo dell'autorità.

Studiosi di architettura e sociologi, tra i quali Marc Augé, Zygmunt Bauman e Rem Koolhass, hanno sostenuto che la struttura della metropoli odierna, caratterizzata peraltro da un processo di uniformazione e disgregazione degli spazi, non permetterebbe quell'uso libero e stimolante del territorio che l'esercizio della flânerie

(55) Réda cerca «les derniers vestiges d'une époque menacée» (H. Micolet, Lire Réda, Lyon, Presses Universitaire de Lyon, 1994, p. 45).

(56) RÉDA, Le Citadin, cit., p. 43.

(57) RÉDA, Châteaux des courants d'air, cit., p. 18.

(58) Ivi, p. 57.

(59) RÉDA, Les Ruines de Paris, cit., p. 76.

(60) Ivi, p. 74.

(61) Ivi, p. 75. In un altro frammento, di fronte a un edificio di recente costruzione che pur cerca di sfuggire al «modèle architectural boîte à chaussures» attraverso un'ostentata originalità, Réda commenta: «C'est une sorte d'hiéroglyphe ou d'idéogramme en stuc, dont l'objet apparent est de célébrer l'inutile» (RÉDA, Châteaux des courants d'air, cit., p. 36).

(62) Il passeggiatore non riesce a distinguere $\mathrm{i}$ confini tra i faubourgs parigini «qui ne veulent jamais finir dans une campagne incapable de commencer» (RÉDA, L'berbe des talus, Paris, Gallimard, 1984 , p. 137).

(63) RÉDA, Châteaux des courants d'air, cit., p. 101.

(64) RÉDA, Les Ruines de Paris, cit., p. 78.

(65) Ivi, p. 44. 
presuppone. Augé, com'è noto, ha suscitato l'attenzione dei sociologi urbani definendo come "non luoghi" ${ }^{65}$ gli spazi che non si integrano alla città storica, che non creano identità né relazione tra gli individui, ma solitudine e similarità. Parcheggi, supermercati, fast food, centri congressi, aeroporti: gli interventi maggiori dell'urbanistica contemporanea non plasmano luoghi ma spazi concepiti per essere attraversati e utilizzati, espressione di un mondo «promis à l'individualité solitaire, au passage, au provisoire et à l'éphémère» ${ }^{67}$. In questo quadro piuttosto desolante, in cui le singolarità delle città storiche convergono verso l'ipotesi di una "città generica", la concretezza della rovina acquisisce un valore irrinunciabile. Laddove l'architettura contemporanea mira a fornire l'illusione di un presente perpetuo e perpetuamente sostituibile - spazi senza identità e senza storia, «qui ne se conjuguent ni au passé ni au futur, sans nostalgie ni espérance» ${ }^{68}$ - le rovine forniscono una testimonianza tangibile dello scorrimento del tempo. In un mondo votato all'oblio di se stesso, pago di un'architettura ibrida e trivialmente spettacolare, le rovine di Parigi sono le "vestali" della storia, isole in cui la corrispondenza tra interiorità del camminatore e forma della città è ancora visibile. In Le temps en ruines, Augé si richiama quindi all'opera di Réda ${ }^{69}$, mettendone in risalto la capacità di portare alla luce attraverso la scrittura le stratificazioni del tempo: elemento che lo distingue da altri scrittori che considerano invece la dimensione temporale della città come un fattore irrilevante rispetto alla sua struttura spaziale.

\section{Scrittura e flânerie, strategie di cancellazione o divertissement}

Se la città appare oggi una superficie liscia e impenetrabile («là où un ciel bleu cru, lavé de tout souvenir, brille indifféremment pour la grâces des femmes et la prospérité du négoce» $)^{70}$, non più "leggibile" come lo era agli occhi del flâneur ottocentesco, essa sembra tuttavia suggerire un nuovo uso, forse più ludico e solipsistico dei suoi spazi. Così, proprio mentre ci racconta la fine della flânerie, mostrandoci il punto di rottura di una tradizione secolare, Réda la mette in scena riaffermandone il valore quale fonte d'ispirazione letteraria e rimedio per vincere lo spleen. Una scelta che si manifesta non tanto nella contemplazione delle "rovine", ma soprattutto di fronte allo spettacolo perennemente rinnovato della città, che suscita curiosità e meraviglia. Ciò dimostra da un lato la continuità con i modelli del passato e, dall'altro, i limiti delle teorie sulla fine ineluttabile della flânerie. Secondo il fisiologista Huart, il flâneur è «le seul homme heureux qui existe sur la terre ${ }^{71}$ : così per Réda «le desespoir n'existe pas pour un homme qui marche» ${ }^{72}$. Il principio di piacere e la libera improvvisazione guidano i suoi spostamenti: «Je dérive, je flâne, je contourne, j'emprunte des raccourcis qui m'égarent et font durer le plaisir» ${ }^{73}$. La flânerie è una squisita tentazione che si offre allo scrittore: «Flâner au long des rues m'écarte [...] de la ligne droite de mon devoir» ${ }^{74}$.

La tendenza alla divagazione, alla fantasticheria, sembra accentuarsi nel corso delle pubblicazioni più recenti, con lo smorzarsi della tensione lirica che caratteriz-

(66) Cfr. M. Augé, Non-lieux, Paris, Édition de Seuil, 1992.

(67) Ivi, p. 101.

(68) Augé, Le temps en ruines, cit., p. 76.

(69) Ivi, p. 89.

(70) RÉDA, La Liberté des rues, cit., p. 53.

(71) Huart, Physiologie du flâneur, cit., p. 11.

(72) RÉDA, Les Ruines de Paris, cit., p. 14.
(73) Ivi, p. 93.

(74) RÉDA, Europes, Saint Clément de rivière, Fata Morgana, 2005, p. 106. Il primo capitolo di La liberté des rues ha come titolo «L'activité du soir». Réda non specifica se si tratti della scrittura o della flânerie, ma entrambe le attività sono considerate come un appuntamento giornaliero, quasi un vizio della vecchiaia. 
zava le prime raccolte. L'attenzione di Réda si focalizza sui dettagli più sfuggenti, inafferrabili come il cielo ${ }^{75}$, mentre la città e le vicende personali dell'autore sono esposte per frammenti, per accumulo e giustapposizione di elementi narrativi. Una scrittura preziosa, calligrafica, divagante, che si esercita non solo nella descrizione del paesaggio parigino, ma anche in occasioni ben diverse, come nel racconto di viaggi o episodi della vita quotidiana dell'autore: «Le goût de la flânerie me semble être une disposition individuelle qui doit trouver à se satisfaire même dans une petite ville ou bien à travers champs» ${ }^{76}$. L'interesse non risiede tanto nell'oggetto o nella storia che si racconta, ma nella disponibilità, nella capacità dello scrittore di rilevarne aspetti sempre nuovi con cui alimentare le proprie fantasticherie. Del resto l'autoironia conduce Réda a non prendere troppo sul serio i propri ragionamenti e l'atto stesso della scrittura $^{77}$, anche quando essi spaziano, come spesso avviene negli ultimi lavori, dal piano fisico a quello metafisico ${ }^{78}$. La scrittura di Réda, autore eccezionalmente prolifico, diventa ora un arabesco, quasi il riempimento di un "vuoto", tanto che i luoghi e le situazioni più banali sono occasione per approfondimenti o digressioni di varia natura:

Je finissais de me raser un matin avec un soin particulier, mais sans m'intéresser plus que d'habitude à l'ensemble de mon reflet dans le miroir (plutôt je m'en garde, car nous nous examinons, lui et moi, avec la stupeur anxieuse de deux être qui, tombés chacun de sa planète, s'effarent moins de leur ressemblance que de la commune monstruosité de leurs traits), quand - mais attendez encore: il faut ouvrir une nouvelle parenthèse à propos de ce don que possèdent les reflets de reproduire avec une exactitude suffocante le moindre détail de ce qui se trouve devant un miroir où ils évoluent comme chez eux, comme nous dans notre monde pourvu de dimensions véritables dont rien ne prouve qu'elles ne présentent pas un avantage trompeur, destiné à nous abuser sur la réalité que nous accordons à nos faits et gestes ${ }^{79}$.

Per Réda, la flânerie non si pratica esclusivamente tra le vie della città. Se Baudelaire aveva stabilito un collegamento tra flânerie e creazione letteraria, vedendo nella prima attività una fonte d'ispirazione per la seconda, Réda sembra portare alle estreme conseguenze questo ragionamento, cimentandosi in una scrittura che diviene essa stessa una divagazione, una flânerie letteraria, un itinerario mentale senza scopo, e perciò inesauribile. Una scrittura che si nutre di se stessa, perché diventata ormai autonoma rispetto alla realtà osservata e descritta dal flâneur. Questa propensione raggiunge il proprio colmo in un frammento dal titolo Une traversée de Paris in cui l'autore, stanco di peregrinare per la città, decide di restare a casa e immaginare un percorso per Parigi che sia un semplice pretesto per la creazione letteraria: «Mais qu'irais-je faire une fois de plus à cette porte mal connue et peu pratiquée, alors que tout me retient devant le clavier de ma machine dont les trentes touches [...] m'autorisent un nombre calculable mais astronomique de fantaisies? $\gg^{80}$.

Anche la scrittura può essere, a suo modo, un mezzo per sfuggire dallo spleen: un vizio solitario, un nobile ed elegantissimo divertissement. «Il arrive qu'à force de marcher $[\ldots .$. , juste en déambulant, on perde peu à peu le sentiment de son identité propre» ${ }^{81}$, aveva scritto Réda in Le citadin: sparire nella folla o nel paesaggio

(75) Cfr. «La modernité, c'est le transitoire, le fugitif, le contingent...» (BAUdelaIRE, Le Peintre de la vie moderne, cit., p 274).

(76) Castigliano, Sept questions à Jacques Réda, cit., p. 14

(77) Cfr. RédA, Ponts flottant, Paris, Gallimard, 2006, p. 94.

(78) In La physique amusante - si cimenta, sulle orme di Lucrezio, a illustrare in versi le teorie della fisica moderna (RÉDA, La Physique amusante, Paris, Gallimard, 2009).

(79) RéDA, Battement, Saint Clément de Rivière, Fata Morgana, 2009, p. 13.

(80) RÉDA, Europes, cit., p. 106.

(81) RÉDA, Le Citadin, cit, p. 11. Il riferimento alla volontà di sparire come soggettività ritorna spesso nei testi di Réda. Ad esempio: «Beaucoup de gens - je ne veux pas m'exclure - vont au café 
che osserva è la vocazione del flâneur, il cui interesse per il mondo fenomenico e la camminata senza meta contrastano rispetto al raccoglimento che l'indagine dell'interiorità postulerebbe. Ora è la scrittura stessa a svolgere questa funzione: una pratica che distrae dal silenzio, che occupa lo spirito altrimenti irrequieto e vuoto, senza che vengano mai meno il rigore e l'impegno formali. L'estro dell'autore si esercita nella cesellatura della frase, nella costruzione architettonica del ragionamento, ricorrendo a una ricca varietà di soluzioni formali, nella prosa come nella poesia. Réda aspira alla frase inalterabile, al cristallo della forma che non vuole sia scalfito dalle insidie dell'interiorità. Programma esistenziale e scrittura sembrano così coincidere: il tono solenne ma distaccato con cui l'autore narra le proprie imprese e il filo onnipresente dell'autoironia segnalano come ogni riferimento concreto e ogni dilemma filosofico che viene suscitato ad arte siano solo un'occasione, quasi un pretesto, per dare spazio al rituale liberatorio della scrittura. Anche le riflessioni metafisiche, gli interessi più disparati di cui Réda dà prova nei testi di recente pubblicazione sono da interpretare secondo questo orientamento: partecipano alla fioritura, alla saturazione verbale che estingue l'borror vacui dello scrittore.

FEDERICO CASTIGLIANO 